\title{
Direct measurement of the charge distribution along a biased carbon nanotube bundle using electron holography
}

Beleggia, Marco; Kasama, Takeshi; Dunin-Borkowski, Rafal E.; Hofmann, S.; Pozzi, G.

Published in:

Applied Physics Letters

Link to article, DOI:

$10.1063 / 1.3598468$

Publication date:

2011

Document Version

Publisher's PDF, also known as Version of record

Link back to DTU Orbit

Citation (APA):

Beleggia, M., Kasama, T., Dunin-Borkowski, R. E., Hofmann, S., \& Pozzi, G. (2011). Direct measurement of the charge distribution along a biased carbon nanotube bundle using electron holography. Applied Physics Letters, 98(24), [243101]. https://doi.org/10.1063/1.3598468

\section{General rights}

Copyright and moral rights for the publications made accessible in the public portal are retained by the authors and/or other copyright owners and it is a condition of accessing publications that users recognise and abide by the legal requirements associated with these rights.

- Users may download and print one copy of any publication from the public portal for the purpose of private study or research.

- You may not further distribute the material or use it for any profit-making activity or commercial gain

- You may freely distribute the URL identifying the publication in the public portal 


\section{$1 D$ Applied Physics \\ Letters}

\section{Direct measurement of the charge distribution along a biased carbon nanotube bundle using electron holography}

M. Beleggia, T. Kasama, R. E. Dunin-Borkowski, S. Hofmann, and G. Pozzi

Citation: Appl. Phys. Lett. 98, 243101 (2011); doi: 10.1063/1.3598468

View online: http://dx.doi.org/10.1063/1.3598468

View Table of Contents: http://apl.aip.org/resource/1/APPLAB/v98/i24

Published by the American Institute of Physics.

\section{Related Articles}

The hysteresis phenomenon of the field emission from the graphene film Appl. Phys. Lett. 99, 173104 (2011)

Temperature dependence of the field emission from the few-layer graphene film Appl. Phys. Lett. 99, 163103 (2011)

Atomic layer coating of hafnium oxide on carbon nanotubes for high-performance field emitters Appl. Phys. Lett. 99, 153115 (2011)

Microstructure evolution and the modification of the electron field emission properties of diamond films by gigaelectron volt Au-ion irradiation

AlP Advances 1, 042108 (2011)

Field emission from hydrogen titanate nanotubes

Appl. Phys. Lett. 99, 143106 (2011)

\section{Additional information on Appl. Phys. Lett.}

Journal Homepage: http://apl.aip.org/

Journal Information: http://apl.aip.org/about/about_the_journal

Top downloads: http://apl.aip.org/features/most_downloaded

Information for Authors: http://apl.aip.org/authors

\section{ADVERTISEMENT}

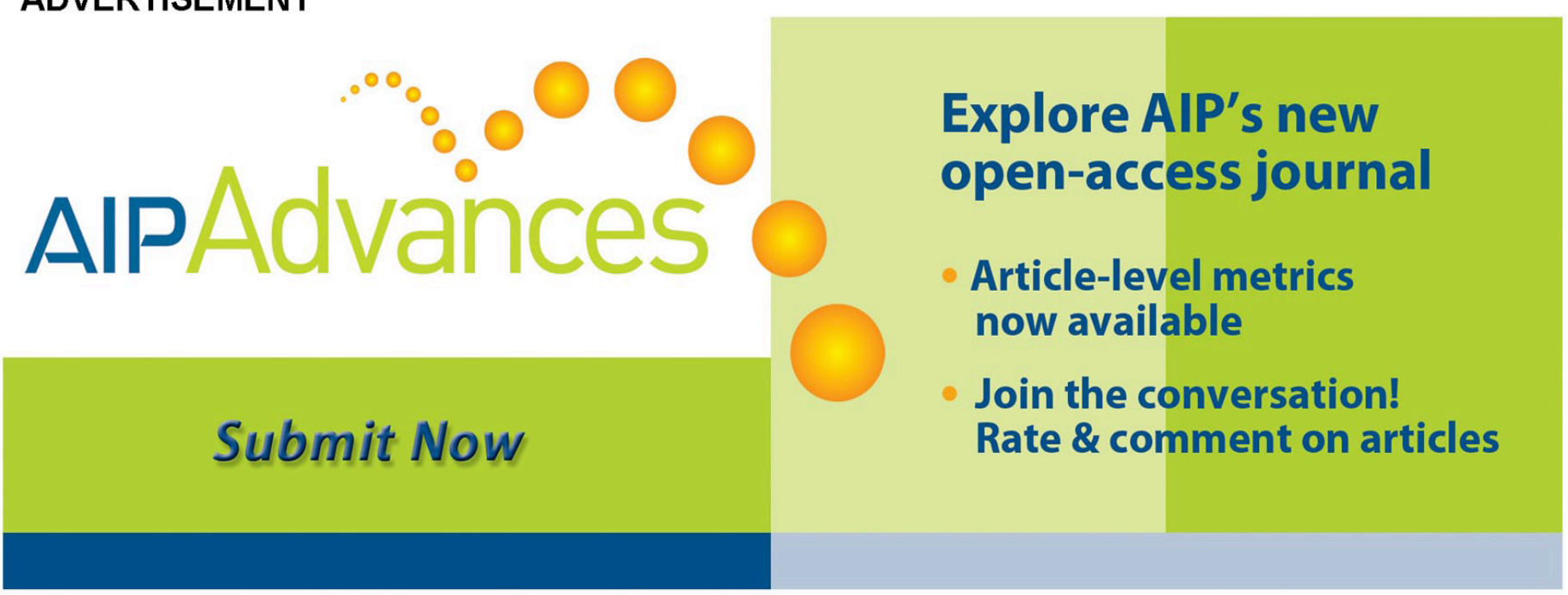




\title{
Direct measurement of the charge distribution along a biased carbon nanotube bundle using electron holography
}

\author{
M. Beleggia, ${ }^{1, a)}$ T. Kasama, ${ }^{1}$ R. E. Dunin-Borkowski, ${ }^{1, b)}$ S. Hofmann, ${ }^{2}$ and G. Pozzi ${ }^{3}$ \\ ${ }^{1}$ Center for Electron Nanoscopy, Technical University of Denmark, 2800 Kongens Lyngby, Denmark \\ ${ }^{2}$ Dept. of Engineering, University of Cambridge, 9 J.J. Thomson Avenue, Cambridge CB3 OFA, \\ United Kingdom \\ ${ }^{3}$ Dipartimento di Fisica, Universitá di Bologna, V.le B. Pichat 6/2, 40127 Bologna, Italy
}

(Received 23 March 2011; accepted 7 April 2011; published online 13 June 2011)

\begin{abstract}
Nanowires and nanotubes can be examined in the transmission electron microscope under an applied bias. Here we introduce a model-independent method, which allows the charge distribution along a nanowire or nanotube to be measured directly from the Laplacian of an electron holographic phase image. We present results from a biased bundle of carbon nanotubes, in which we show that the charge density increases linearly with distance from its base, reaching a value of $\sim 0.8$ electrons/nm near its tip. (C) 2011 American Institute of Physics.

[doi:10.1063/1.3598468]
\end{abstract}

An important technological application of high aspect ratio nanostructures, in particular carbon nanotubes (CNTs), is their use in field emission cathodes, e.g., for compact X-ray sources, ${ }^{1}$ microwave amplifiers, ${ }^{2}$ and electron sources for microscopy. ${ }^{3}$ Here we examine bundles of single-walled CNTs (SWNTs), which extend from a grounded conducting substrate, under an applied bias in the transmission electron microscope (TEM). The applied electric field results in charge being transferred onto the SWNT bundle to maintain its surface equipotential. The theoretical prediction of the magnitude and distribution of charge along a high-aspect ratio nanostructure is a difficult problem, whose solution lies at the interface between classical electrostatics ${ }^{4-6}$ and quantum theory in condensed matter physics. ${ }^{7-10}$ We use off-axis electron holography, ${ }^{11-13}$ a technique that has previously been applied to visualize the trend of the projected electrostatic potential around charged objects, ${ }^{14-16}$ to show that the distribution of charge along a SWNT bundle can be determined experimentally with nanometer spatial resolution without prior knowledge about the geometry of the specimen and model independently.

Electrical biasing experiments were carried out in a Philips CM300 field emission gun TEM equipped with an electron biprism and a Lorentz lens. A schematic diagram of the setup for the TEM mode of off-axis electron holography is shown in Fig. 1(a). As a sample we used SWNT bundles extending from the roughened edges of purified buckypaper. A specialized TEM specimen holder ${ }^{17}$ was used to place the sample onto a fixed electrical contact with a gold needle used as a movable counterelectrode that could be placed at a chosen distance from the selected SWNT bundle. Off-axis electron holograms (and vacuum reference holograms) were acquired at $300 \mathrm{kV}$ in Lorentz mode with the conventional objective lens switched off, in order to provide an optimal field of view and interference fringe spacing. A biprism voltage of $200 \mathrm{~V}$ was used, resulting in a field of view in each hologram of $840 \mathrm{~nm}$ and an interference fringe spacing of $2.2 \mathrm{~nm}$. Figures 1(b) and 1(c) show highly defocused images

\footnotetext{
${ }^{a)}$ Electronic mail: mb@cen.dtu.dk.

${ }^{b}$ Present address: Institute for Microstructure Research, Forschungszentrum Jülich, 52425 Jülich, Germany.
}

of a chosen region of SWNT bundles examined under an applied bias of $50 \mathrm{~V}$ with a gold counterelectrode placed at a distance of $\sim 3.6 \mu \mathrm{m}$ from the tips of the bundles. The change in contrast exhibited by each bundle along its length results from its charge density distribution.

The electrostatic contribution to the phase shift of a high-energy electron wave recorded using electron holography is given by the expression

$$
\varphi(x, y)=C_{E} \int_{-\infty}^{+\infty} V(x, y, z) \mathrm{d} z
$$

where $V(x, y, z)$ is the electrostatic potential within and around the specimen, $C_{E}$ is a constant that depends on the microscope accelerating voltage $\left(C_{E}=6.526 \mathrm{rad} \mathrm{V}^{-1} \mu \mathrm{m}^{-1}\right.$ at $300 \mathrm{kV}$ ), and $z$ is the incident electron beam direction.

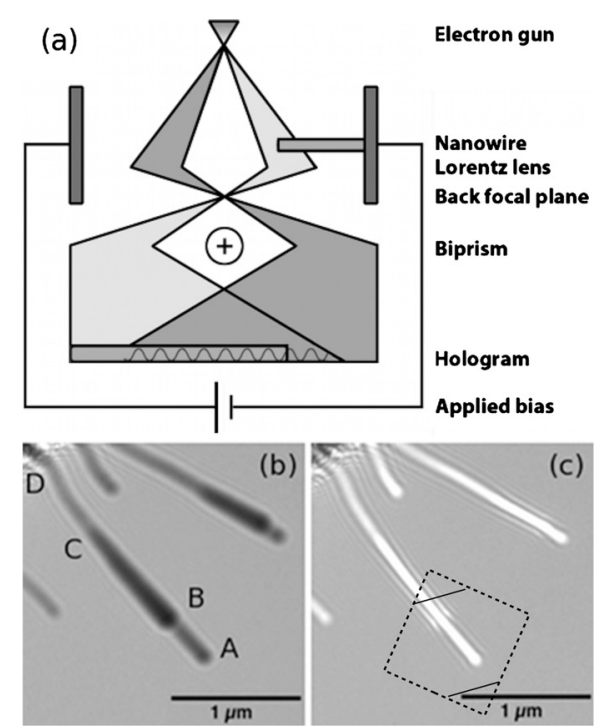

FIG. 1. (a) Schematic diagram of the setup used to record off-axis electron holograms in the TEM. (b) and (c) Defocused images acquired from biased SWNT bundles placed $3.6 \mu \mathrm{m}$ from a Au counterelectrode. The bias applied is $50 \mathrm{~V}$. Image (b) is acquired $\sim 3 \mathrm{~mm}$ overfocus while image (c) is acquired $\sim 3 \mathrm{~mm}$ underfocus. Letters A-D in (b) mark significant locations along the nanowire while the dashed box in (c) indicates the region from where the phase image in Fig. 2(a) was acquired. 


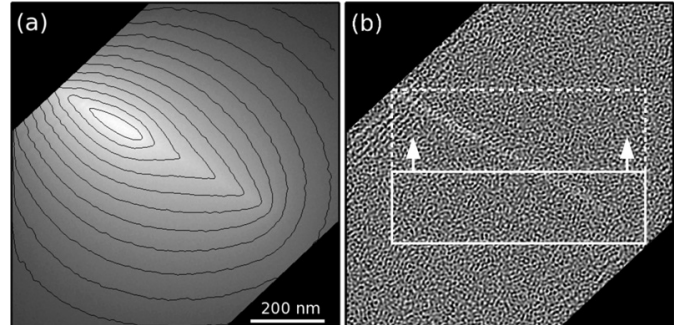

FIG. 2. (a) Phase shift measured using off-axis electron holography from the square region marked in Fig. 1(b) for an applied electrical bias between the nanowire and the counterelectrode of $50 \mathrm{~V}$. The contour spacing is $1.57 \mathrm{rad}$. (b) Laplacian of (a). The annotations in (b) indicate the rectangular regions used to determine the cumulative charge along the wire.

The theoretical basis of the proposed approach follows directly from the laws of electrostatics. The electrostatic potential generated by a time-independent volume charge distribution $\rho(x, y, z)$ obeys Poisson's equation

$$
\nabla^{2} V(x, y, z)=-\frac{\rho(x, y, z)}{\epsilon_{0}} .
$$

If the electric field vanishes at infinity, then we can combine Eqs. (1) and (2) to establish the proportionality relation

$$
\nabla^{2} \varphi(x, y)=-\frac{C_{E}}{\epsilon_{0}} \sigma_{p}(x, y)
$$

between the two-dimensional Laplacian of the phase and the projected charge density

$$
\sigma_{p}(x, y)=\int_{-\infty}^{+\infty} \rho(x, y, z) d z
$$

According to Eq. (3), the Laplacian of the phase shift is a representation of the projected charge density $\sigma_{p}(x, y)$ at all points across the field of view. In order to measure the cumulative charge along the length of the SWNT bundle, we integrate the Laplacian of the phase over regions $\mathcal{D}$ that include increasing portions of the bundle, in the form

$$
\begin{aligned}
Q_{\mathcal{D}} & =-\frac{\epsilon_{0}}{C_{E}} \iint_{\mathcal{D}} \nabla^{2} \varphi(x, y) d x d y=\iint_{\mathcal{D}} \sigma_{p}(x, y) d x d y \\
& =\int_{-\infty}^{\infty} d z \iint_{\mathcal{D}} \rho(x, y, z) d x d y .
\end{aligned}
$$

This charge is, by definition, the volume integral of the charge distribution over the region considered, or, according to Eq. (2), the volume integral of the three-dimensional Laplacian of the electrostatic potential over the same region. One of the three integrals is effectively carried out by recording the phase image while the two remaining integrals are performed numerically over suitable portions of the twodimensional Laplacian of the phase shift.

In order to bypass several image processing steps, including phase unwrapping, we make use of complex algebra to obtain the Laplacian of the phase directly from the reconstructed complex image wave $\psi=a \exp (\mathrm{i} \varphi)$ by using

$$
\nabla^{2} \varphi=\operatorname{Im}\left[\frac{\nabla^{2} \psi}{\psi}-\frac{(\nabla \psi)^{2}}{\psi^{2}}\right]
$$

Figure 2(a) shows the phase shift recorded from the boxed region in Fig. 1(b), with $\pi / 2$ rad equiphase contours

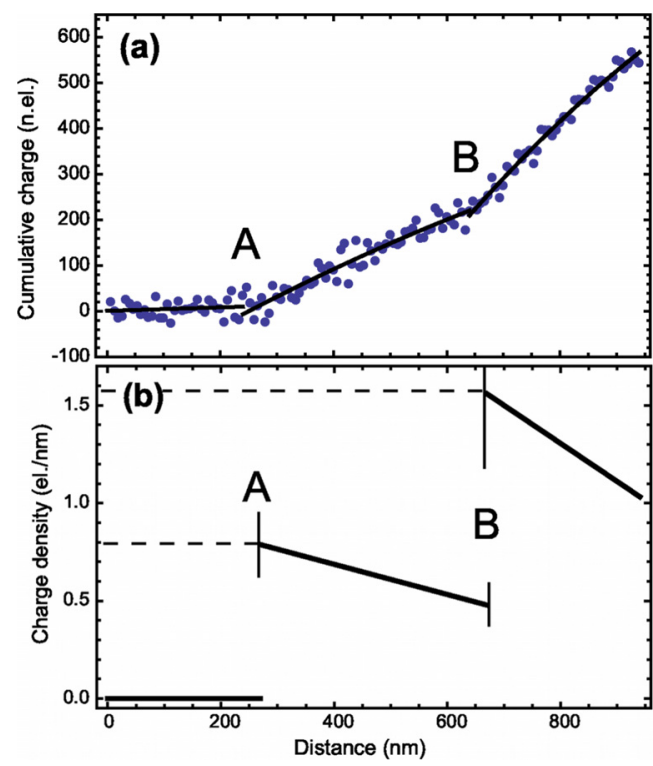

FIG. 3. (Color online) (a) Cumulative charge and (b) inferred local charge density profiles along the visible portion of the SWNT bundle. In (a), each dot corresponds to the Laplacian of the phase integrated over a rectangle of increasing size [see Fig. 2(b)] while the lines correspond to polynomial fits to the data. In (b), the vertical error bars represent the uncertainty in the measured charge density at positions A and B [see Fig. 1(b)].

superimposed, while Fig. 2(b) shows the Laplacian of Fig. 2(a) calculated according to Eq. (6). In order to measure the charge density along the bundle, we integrate the Laplacian of the phase within rectangles of increasing height, as sketched in Fig. 2(b). After taking into account the inclination of the bundle with respect to the direction along which the integration is performed, we obtain the cumulative charge profile shown in Fig. 3(a), in which two slopes are visible. In the out-of-focus images shown in Figs. 1(b) and 1(c), the contrast changes at two positions along the structure, labeled B and C in Fig. 1(b), between which it appears to change diameter locally. The charge density along the bundle therefore appears to be in three distinct sections; from the support D to C, from $\mathrm{C}$ to $\mathrm{B}$, and from $\mathrm{B}$ to the tip A.

After fitting the measured cumulative charge profile in each region of the bundle using a second order polynomial, we differentiate the fitted profiles to obtain a measure of the charge density along its length. In the resulting graph shown in Fig. 3(b), the charge density can be seen to reach a value of $(0.79 \pm 0.18) \mathrm{e} / \mathrm{nm}$ near the tip (position A). It decreases linearly to $(0.48 \pm 0.11) \mathrm{e} / \mathrm{nm}$ at a distance of $400 \mathrm{~nm}$ from the tip (position B) at a rate of $(7.7 \pm 2.5) \times 10^{-4} \mathrm{e} / \mathrm{nm}^{2}$ and then increases abruptly to $(1.6 \pm 0.4) \mathrm{e} / \mathrm{nm}$, subsequently decreasing back to $(1.0 \pm 0.3) \mathrm{e} / \mathrm{nm}$ at the edge of the field of view at a distance of $\sim 670 \mathrm{~nm}$ from the tip at a rate of $(20 \pm 8) \times 10^{-4} \mathrm{e} / \mathrm{nm}^{2}$. The uncertainties are determined from the standard deviations of the polynomial fits and are relatively large due to the limited number of data points. Based on the contrast in Figs. 1(b) and 1(c), for the portion of the bundle that is outside the field of view we assume a linear trend of the charge density to position C. At this position, we assume that the charge density reverts to the same trend that it had near the tip, reaching zero at the point of contact $\mathrm{D}$ with the substrate $(\sim 2 \mu \mathrm{m}$ from $\mathrm{A})$.

As a self-consistency check, the measured and extrapolated charge densities were used to simulate the phase shift of a charged wire of the same length and negligible radius 

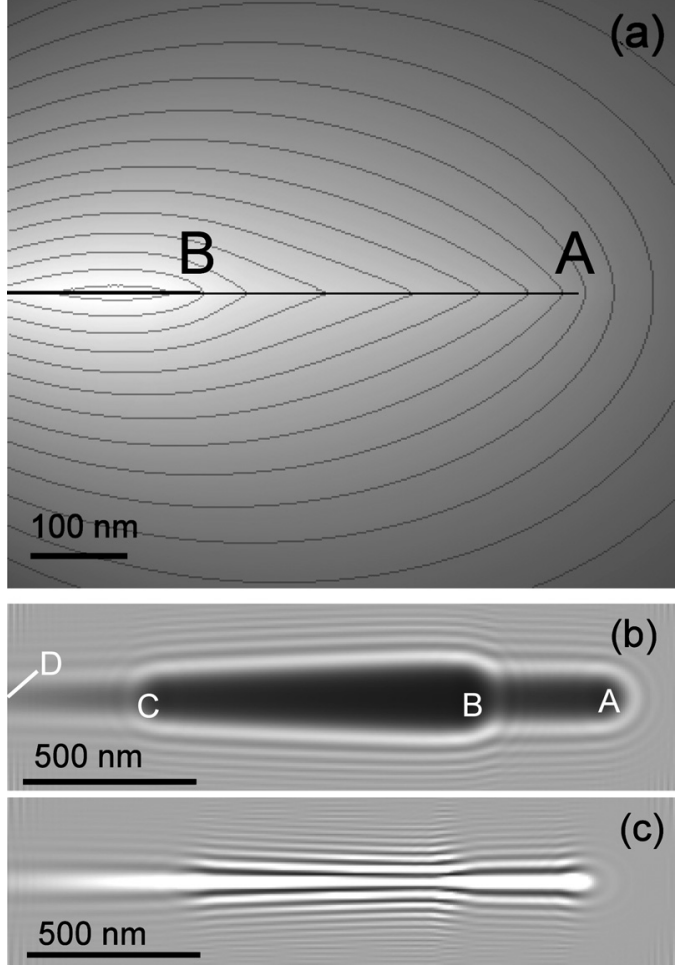

FIG. 4. (a) Simulated phase image generated by using a superposition of the terms described in Eq. (7), for the measured charge densities; the spacing of the phase contours is $1.57(\pi / 2)$ rad. (b) and (c) corresponding simulations of defocused bright-field images of the same nanowire for defocus values of $\pm 3 \mathrm{~mm}$, for comparison with Figs. 1(b) and 1(c). In (b) and (c) the image intensity is displayed between values of 0 and 1.5 .

attached to a conducting substrate. The calculations were performed by making use of the expression for the phase shift of a wire in which the charge density $\lambda(x)=\sigma_{w} x$ is linear, where $\sigma_{w}$ is a constant with the same dimensions as a surface charge density. For a wire that has its end-points at $x=0$ and $x=L$, the phase shift can be calculated by means of standard definite integrals and is given by the expression

$$
\begin{aligned}
\varphi(x, y)= & \frac{\sigma_{w} C_{E}}{8 \pi \epsilon_{0}}\left[\left(L^{2}-x^{2}+y^{2}\right) \log \frac{(x+L)^{2}+y^{2}}{(x-L)^{2}+y^{2}}+4 L x\right. \\
& \left.+4 x y \arctan \left(\frac{x-L}{y}\right)-4 x y \arctan \left(\frac{x+L}{y}\right)\right] .
\end{aligned}
$$

By making use of Eq. (7), the measured charge densities at $\mathrm{A}$ and $\mathrm{B}$ and the estimated lengths of the intervals $\mathrm{AB}$ ( $\sim 400 \mathrm{~nm}), \mathrm{BC}(\sim 900 \mathrm{~nm})$, and CD $(\sim 730 \mathrm{~nm})$, we simulated a phase image and corresponding defocused bright-field TEM images for comparison with Figs. 2(a), 1(b), and 1(c), respectively. While subtle differences are visible between the simulations shown in Fig. 4 and the experimental data, possibly caused by neglecting partial coherence of the illumination and spherical aberration, the similarity is encouraging. If we use the charge distribution to estimate the electric field in the proximity of the tip, we obtain a value of $\sim 0.22 \mathrm{~V} / \mathrm{nm}$ at a distance of $5 \mathrm{~nm}$, decreasing to $\sim 0.02 \mathrm{~V} / \mathrm{nm}$ at a distance of $50 \mathrm{~nm}$. The axial component of the electric field in the tip region follows the simple law $E_{x}=V_{t} / d$ for several tens of nanometers, where $V_{t} \sim 1.1 \mathrm{~V}$ and $d$ is the distance from the tip. More extensive simulations, involving variations in the parameters within the experimental uncertainties, could be used to quantify the electric field and potential at all points outside the SWNT bundle.
Significantly, our approach is independent of the distribution of image charges in the support, so long as the integration loop does not enclose regions in which the image charges are present. In addition, if the vacuum reference wave that is used to form the hologram is perturbed by charges that are present either within the structure or outside the field of view, then the measurement of the charge within the object of interest is not affected, unlike the phase image itself. By the same token, neither any electric field that may influence the reference hologram nor any linear phase ramps affect the measured charge. The method is therefore not only able to provide the charge distribution but it is also, in principle, unaffected by several artifacts that usually influence electron holographic phase images. ${ }^{18}$

A note of caution is required with regard to the effect of the mean inner potential (MIP) on such measurements. Since no net charge is associated with the MIP the measurement of the total charge over a region that includes the entire object is independent of the MIP. However, the MIP contribution to the local phase shift of an uncharged object does produce an effective projected charge distribution that prevents us from being able to interpret $\sigma_{p}(x, y)$ [shown here in Fig. 2(b)] as originating exclusively from physical charges inside the object. In the present example, the MIP contribution to $\sigma_{p}(x, y)$ can be ignored due to the small diameter of the nanowire. However, in general, one needs to consider its possible influence, as well as that of undersampling.

We envision a broad range of scenarios to which the method may be extended, including the measurement of charge density distributions associated with dopant potentials in semiconductors and charges within insulators. Future studies will include detailed comparisons of measured charge densities in nanowires and nanotubes with variations in their morphology, chemical composition, and microstructure.

${ }^{1}$ X. Calderón-Colón, H. Geng, L. An, G. Cao, and O. Zhou, Nanotechnology 20, 325707 (2009)

${ }^{2}$ K. B. K. Teo, E. Minoux, L. Hudanski, F. Peauger, J.-P. Schnell, L. Gangloff, P. Legangneux, D. Dieumegard, G. A. J. Amaratunga, and W. I. Milne, Nature (London) 437, 968 (2005).

${ }^{3}$ N. de Jonge, Adv. Imaging Electron Phys. 156, 203 (2009).

${ }^{4}$ G. C. Kokkorakis, A. Modinos, and J. P. Xanthakis, J. Appl. Phys. 91, 4580 (2002).

${ }^{5}$ D. G. Walker, W. Zhang, and T. S. Fisher, J. Vac. Sci. Technol. B 22, 1101 (2004).

${ }^{6}$ L. D. Filip, D. Nicolaescu, M. Tanemura, S. Kanemaru, and J. Itoh, J. Vac. Sci. Technol. B 23, 649 (2005).

${ }^{7}$ S. Han, M. H. Lee, and J. Ihm, Phys. Rev. B 65, 085405 (2002).

${ }^{8}$ X. Zheng, G. Chen, Z. Li, S. Deng, and N. Xu, Phys. Rev. Lett. 92, 106803 (2004).

${ }^{9}$ L. H. Pan, V. J. Peridier, and T. E. Sullivan, Phys. Rev. B 71, 035345 (2005).

${ }^{10}$ T. M. Marinov and S. I. Hariharan, J. Appl. Phys. 105, 064308 (2009).

${ }^{11}$ A. Tonomura, Electron Holography, 2nd ed. (Springer, Berlin, Heidelberg, 1999).

${ }^{12}$ Introduction to Electron Holography, edited by E. Völk1, L. F. Allard, and D. C. Joy (Kluwer Academic, New York, 1999).

${ }^{13}$ H. Lichte and M. Lehmann, Rep. Prog. Phys. 71, 016102 (2008).

${ }^{14}$ J. Cumings, A. Zettl, M. R. McCartney, and J. C. H. Spence, Phys. Rev. Lett. 88, 056804 (2002).

${ }^{15}$ L. J. Chou, M. T. Chang, Y. L. Chueh, J. J. Kim, H. S. Park, and D. Shindo, Appl. Phys. Lett. 89, 023112 (2006).

${ }^{16}$ B. Cho, T. Ishikawa, and C. Oshima, Appl. Phys. Lett. 91, 163102 (2007).

${ }^{17}$ R. E. Dunin-Borkowski et al., MRS Symposia Proceedings No. 907E (Materials Research Society, Pittsburgh, 2005), p. MM13.02.

${ }^{18}$ G. Matteucci, G. F. Missiroli, and G. Pozzi, Adv. Imaging Electron Phys 122, 173 (2002). 